\title{
Adenocarcinoma indiferenciado como factor predictor de metástasis ganglionares en cáncer gástrico
}

\author{
Undifferentiated adenocarcinoma as a predictor of lymph node \\ metastases in gastric cancer
}

Ana María García1', William Sánchez²

1 Médica, residente de Cirugía general, Hospital Militar Central, Universidad Militar Nueva Granada, Bogotá, D.C., Colombia.

2 Médico, especialista en Cirugía general, jefe del Departamento de Cirugía General, Hospital Militar Central, Bogotá, D.C., Colombia.

\section{Resumen}

Introducción. El grado de diferenciación celular en el estudio histopatológico del adenocarcinoma gástrico está descrito como un factor pronóstico determinante en el comportamiento clínico del tumor. El adenocarcinoma gástrico indiferenciado es considerado una variante agresiva de mal pronóstico, que se correlaciona con una alta tasa de metástasis ganglionares.

Métodos. Estudio prospectivo descriptivo de una serie de casos en el cual se analizan los pacientes con adenocarcinoma gástrico indiferenciado, que fueron llevados a cirugía radical con gastrectomía y linfadenectomía DII y su correlación con la presencia de metástasis ganglionares en un período de dos años.

Resultados. De enero de 2018 a enero de 2020 se recolectaron en la base de datos 113 pacientes con adenocarcinoma gástrico a quienes se les practicó gastrectomía total, disección ganglionar DII y reconstrucción esofagoyeyunal terminolateral con técnica de Orr más Y de Roux. Fueron clasificados histológicamente como adenocarcinoma gástrico indiferenciado 41 pacientes (36,3\%). La edad promedio de este grupo fue de 56 años con un rango entre 28-92 años. De ellos 30 fueron hombres (73\%) y 11 mujeres (27\%). El número promedio de ganglios linfáticos analizados por espécimen fue de 24. De los 41 pacientes con adenocarcinoma gástrico indiferenciado, 35 (85 \%) tuvieron metástasis ganglionares, con 382 ganglios positivos en total, con un rango entre 1-38 y un promedio de 11 ganglios linfáticos positivos por espécimen.

Discusión. En esta serie el adenocarcinoma gástrico indiferenciado se presentó en el 36,3 \% de los casos y se correlacionó con un 85 \% de presencia de metástasis ganglionares en estadios T3-T4.

Palabras clave: cáncer gástrico; adenocarcinoma; diferenciación celular; metástasis ganglionares; pronóstico.

Fecha de recibido: 15/09/2020 - Fecha de aceptación: 4/03/2021 - Fecha de publicación en línea: 23/07/2021

Correspondencia: William Sánchez, transversal 3a \# 49-00 Jefatura Servicio de Cirugía general, Hospital Militar Central, Bogotá D.C., Colombia. Teléfono: 315 3322811. Correo electrónico: sanchez.william@cable.net.co

Citar como: García AM, Sánchez W. Adenocarcinoma indiferenciado como factor predictor de metástasis ganglionares en cáncer gástrico. Rev Colomb Cir. 2021;36:620-5. https://doi.org/10.30944/20117582.860

Este es un artículo de acceso abierto bajo una Licencia Creative Commons - BY-NC-ND https://creativecommons.org/licenses/by-ncnd/4.0/deed.es 


\begin{abstract}
Introduction. The degree of cellular differentiation in the histopathological study of gastric adenocarcinoma is described as a determining prognostic factor in the clinical behavior of the tumor. Undifferentiated gastric adenocarcinoma is considered an aggressive variant with a poor prognosis, which is correlated with a high rate of lymph node metastasis.
\end{abstract}

Methods. Descriptive prospective study of a series of cases in which patients with undifferentiated gastric adenocarcinoma who underwent radical surgery with DII gastrectomy and lymphadenectomy and their correlation with the presence of lymph node metastases in a period of two years.

Results. From January 2018 to January 2020,113 patients with gastric adenocarcinoma were collected in the database who underwent total gastrectomy, DII lymph node dissection and end-to-side esophagojejunal reconstruction with the Orr plus Roux-en-Y technique. Forty-one patients (36.3\%) were histologically classified as undifferentiated gastric adenocarcinoma. The average age of this group was 56 years with a range between 28-92 years. Of these, 30 were men (73\%) and 11 women (27\%). The mean number of lymph nodes analyzed per specimen was 24 . Of the 41 patients with undifferentiated gastric adenocarcinoma, 35 (85\%) had lymph node metastases, with 382 positive nodes in total, with a range between 1-38 and a mean of 11 positive lymph nodes per specimen.

Discussion. In this series, undifferentiated gastric adenocarcinoma occurred in $36.3 \%$ of cases and was correlated with $85 \%$ of the presence of lymph node metastases in T3-T4.

Keywords: gastric cancer; adenocarcinoma; cell differentiation; lymph node metastases; prognosis.

\section{Introducción}

La cirugía continúa siendo la única modalidad terapéutica con potencial intención curativa en el tratamiento del adenocarcinoma gástrico (ACG) ${ }^{1}$. A pesar de los últimos avances en genética y biología molecular, que han permitido un mejor entendimiento de la carcinogénesis de esta enfermedad, y del desarrollo de tratamientos neoadyuvantes y adyuvantes, los dos principales factores predictores biológicos del comportamiento de la enfermedad continúan siendo el grado de penetración del tumor a través de la pared y el estadio ganglionar, expresados en la clasificación TNM, aún vigente ${ }^{1,2}$.

Basados en el análisis histológico, el ACG se ha clasificado tradicionalmente en diferenciado e indiferenciado. Este último tipo histológico se ha considerado como un factor de riesgo independiente para la presencia de metástasis ganglionares $(\mathrm{MG})^{1,3}$. En los tumores intramucosos, las MG se presentan entre el 4,2 - 6,0 \% y su frecuencia va aumentando hasta el 40 - $60 \%$ en los tumores más avanzados, asociado con otros factores pronósticos, como el tamaño tumoral, la infiltración linfovascular y la ulceración ${ }^{4,5}$.
En Colombia, debido a la ausencia de programas de tamización a gran escala para ACG, la mayoría de los diagnósticos se hacen en etapas avanzadas que exigen un gran esfuerzo terapéutico, en el que la gastrectomía con linfadenectomía continúa siendo el tratamiento quirúrgico estándar para el adenocarcinoma gástrico indiferenciado (ACGI) ${ }^{1,6}$. El objetivo de este estudio fue analizar, de forma prospectiva y por un periodo de dos años, a los pacientes con ACGI y su correlación con la presencia de MG, para determinar las implicaciones terapéuticas subsecuentes.

\section{Métodos}

Estudio prospectivo de una serie de casos de pacientes con ACG que fueron atendidos en la Unidad de Cirugía Oncológica del Hospital Militar Central, en Bogotá D.C., Colombia, entre enero de 2018 y enero de 2020, y se les practicó gastrectomía total con linfadenectomía, según los criterios establecidos por la Japanese Research Society for Gastric Cancer, considerados a nivel mundial actualmente como el patrón de oro para el manejo quirúrgico ${ }^{7}$. Se incluyeron pacientes mayores de 18 años, sin cirugías gástricas previas, con diagnóstico histo- 
lógico confirmado de adenocarcinoma gástrico. El ACGI se basó en los criterios histológicos establecidos por Brothers y posteriormente modificados por Nakamura $3,8,9$.

La recolección de datos y el análisis estadístico se realizó con el sistema SPSS; se aplicó la prueba de Chi cuadrado y se consideró una p significativa al valor inferior a 0,05.

\section{Resultados}

En el periodo de estudio se recolectaron en la base de datos 113 pacientes con adenocarcinoma gástrico que fueron llevados a gastrectomía total (Figura 1), disección ganglionar DII y reconstrucción esofagoyeyunal terminolateral con técnica de Orr + Y de Roux (Figura 2). Se excluyeron del estudio 72 pacientes $(63,7 \%)$ con diagnóstico histológico de adenocarcinoma gástrico bien o moderadamente diferenciados. Fueron clasificados histológicamente como adenocarcinoma indiferenciado e incluidos en el estudio 41 pacientes (36,3 \%) (Figura 3). La edad promedio de este grupo fue de 56 años, con un rango entre 28 a 92 años. Correspondieron a hombres 30 casos (73\%)

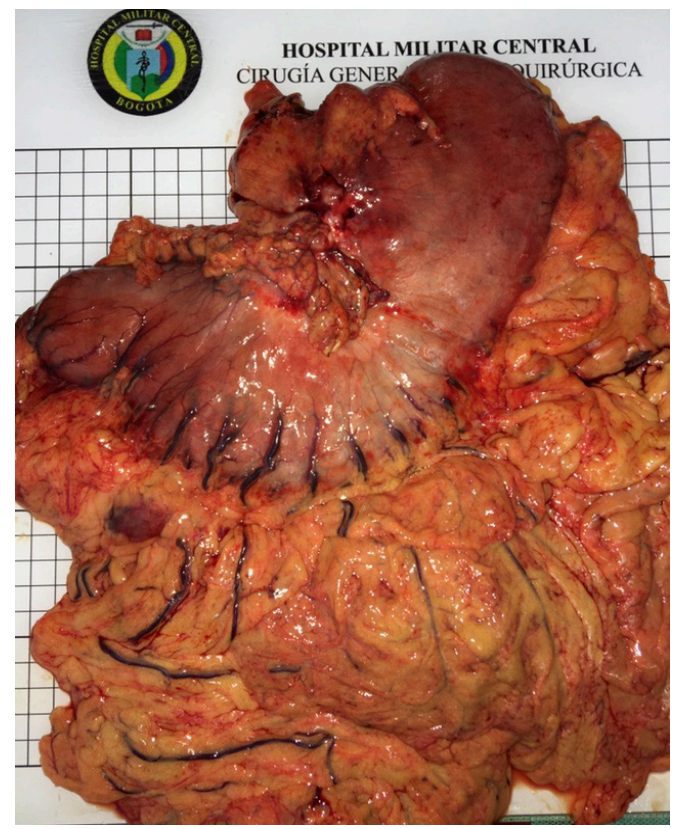

Figura 1. Pieza quirúrgica de gastrectomía total. y a mujeres 11 (27\%). En relación con el grado de penetración del tumor a través de la pared gástrica, se encontraron los siguientes resultados: $\mathrm{T} 1=1$; $\mathrm{T} 2=5$; T3=27 y T4=8. Es decir que el $85 \%$ de los pacientes con ACGI $(n=35)$ presentaban tumores localmente avanzados (Tabla1).

El total de ganglios linfáticos analizados fue de 969, con un rango entre 8-40 y un promedio de ganglios linfáticos analizados por espécimen de 24. De los pacientes con ACGI, $85 \%$ (n=35) tuvieron metástasis ganglionares, con 382 ganglios positivos en total, con un rango entre 1 - 38 y un promedio de 10,9 ganglios linfáticos positivos por espécimen. De los seis pacientes con ACGI que no presentaron metástasis ganglionar, uno tenía una lesión T1, otro una lesión T2 y cuatro no tenían invasión linfovascular.

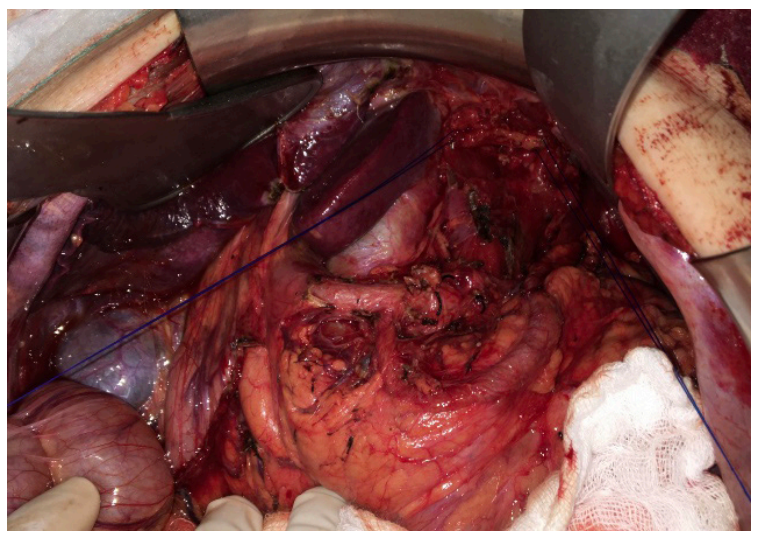

Figura 2. Imagen intraoperatoria de linfadenectomía D2.

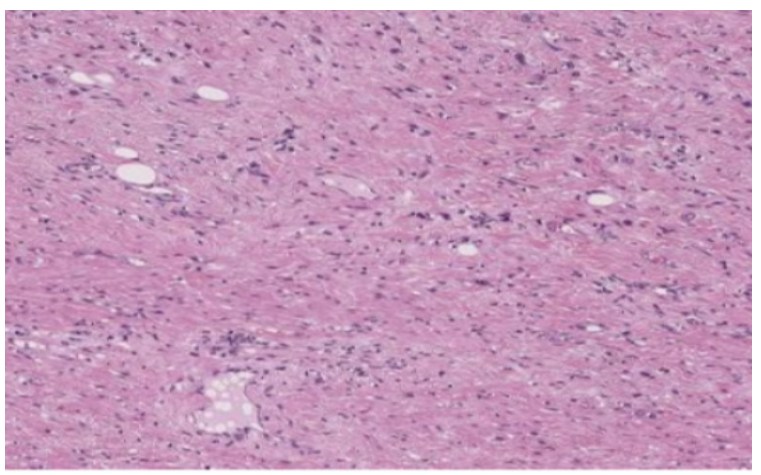

Figura 3. Adenocarcinoma gástrico indiferenciado con intensa desmoplasia. Hematoxilina \& eosina. 10x 
Tabla 1. Pacientes con adenocarcinoma gástrico indiferenciado, estadio tumoral $\mathrm{T}$, total de ganglios linfáticos resecados y total de ganglios linfáticos metastásicos $(n=41)$

\begin{tabular}{|c|c|c|c|c|}
\hline Sexo & Edad & grado $\mathrm{T}$ & Ganglios & Positivos \\
\hline Masculino & 33 & T3 & 36 & 8 \\
\hline Masculino & 30 & T3 & 39 & 35 \\
\hline Masculino & 92 & $\mathrm{~T} 2$ & 13 & 10 \\
\hline Masculino & 61 & T3 & 13 & 10 \\
\hline Masculino & 60 & T3 & 18 & 5 \\
\hline Masculino & 74 & $\mathrm{~T} 4$ & 24 & 27 \\
\hline Masculino & 76 & T3 & 20 & 6 \\
\hline Masculino & 79 & $\mathrm{~T} 4$ & 35 & 35 \\
\hline Masculino & 74 & T3 & 23 & 12 \\
\hline Masculino & 39 & T3 & 28 & 21 \\
\hline Masculino & 87 & T3 & 17 & 3 \\
\hline Masculino & 78 & T3 & 27 & 22 \\
\hline Masculino & 68 & T3 & 14 & 3 \\
\hline Masculino & 85 & T3 & 24 & 3 \\
\hline Masculino & 75 & $\mathrm{~T} 4$ & 27 & 16 \\
\hline Masculino & 32 & T3 & 19 & 2 \\
\hline Masculino & 72 & $\mathrm{~T} 2$ & 19 & 1 \\
\hline Masculino & 45 & $\mathrm{~T} 2$ & 33 & 8 \\
\hline Masculino & 40 & T2 & 20 & 11 \\
\hline Masculino & 33 & T3 & 15 & 11 \\
\hline Masculino & 31 & $\mathrm{~T} 4$ & 20 & 20 \\
\hline Masculino & 65 & T3 & 32 & 2 \\
\hline Masculino & 69 & T3 & 15 & 6 \\
\hline Masculino & 60 & T3 & 27 & 1 \\
\hline Masculino & 63 & T3 & 28 & 16 \\
\hline Femenino & 70 & $\mathrm{~T} 4$ & 38 & 5 \\
\hline Femenino & 63 & T3 & 14 & 2 \\
\hline Femenino & 31 & T3 & 19 & 5 \\
\hline Femenino & 46 & T3 & 33 & 4 \\
\hline Femenino & 58 & T3 & 40 & 2 \\
\hline Femenino & 61 & $\mathrm{~T} 4$ & 25 & 17 \\
\hline Femenino & 44 & T3 & 17 & 10 \\
\hline Femenino & 88 & T3 & 9 & 3 \\
\hline Femenino & 68 & T3 & 12 & 2 \\
\hline Femenino & 69 & $\mathrm{~T} 4$ & 40 & 38 \\
\hline Masculino & 71 & T3 & 19 & 0 \\
\hline Femenino & 28 & $\mathrm{~T} 4$ & 20 & 0 \\
\hline Masculino & 69 & T3 & 35 & 0 \\
\hline Masculino & 44 & T3 & 29 & 0 \\
\hline Masculino & 37 & $\mathrm{~T} 2$ & 25 & 0 \\
\hline Masculino & 36 & $\mathrm{~T} 1$ & 8 & 0 \\
\hline
\end{tabular}

\section{Discusión}

Brothers y posteriormente Nakamura dieron a conocer e hicieron global el concepto del grado de diferenciación histológica del adenocarcinoma gástrico como un factor histológico de importancia relevante, que puede predecir cómo será el comportamiento a futuro del tumor y su correlación con la presencia de metástasis ganglionares, las cuales siguen siendo el principal factor predictor de supervivencia global y de libre de enfermedad en los análisis univariados y multivariados para ACG $^{3,8-10}$.

Fenotípicamente, el ACGI tiene particularidades clínicas diferenciales. Generalmente son lesiones proximales, de comportamiento difuso y ulceradas, asociadas a tumores con células en anillo de sello y muy alta tasa de metástasis ganglionares ${ }^{11,12}$. Estudios de biología molecular han podido correlacionar diferentes factores pronósticos adversos relacionados con tumores indiferenciados gástricos, por ejemplo, la baja expresión de las proteínas P27kip1 y P21cip1, que son ciclinas dependientes de las quinasas directamente relacionadas al funcionamiento del gen p53, que se identifica en la progresión de la carcinogénesis con una disminución progresiva de una mucosa gástrica normal a una gastritis superficial crónica y su progresión a lesiones precancerosas o invasivas avanzadas, sugiriendo que la perdida de la expresión de la proteína P21 está estrechamente relacionada con la perdida de la diferenciación histológica del tumor, incrementando su potencial de invasión tumoral, favoreciendo la presencia de enfermedad metastásica ganglionar y a distancia ${ }^{13,14}$.

En el tratamiento del cáncer gástrico temprano mediante mucosectomía o resección endoscópica segmentaria, la identificación de un ACGI en la patología es crucial para la selección del tratamiento a realizar. En lesiones tumorales que solo afectan la mucosa, las metástasis ganglionares se han encontrado entre el 4,2 - $6 \%$ y se incrementan al $11-23,8 \%$ en las lesiones que penetran la submucosa y que están acompañadas de factores adversos como un tamaño mayor a $2 \mathrm{~cm}$, infiltración linfovascular presente y ulceración. 
Sin embargo, aún en los tumores tempranos bien diferenciados, las metástasis ganglionares se pueden presentar entre el $0,4-1,8 \%$, lo que permite concluir que el proceso de diseminación metastásica ganglionar no es dependiente de un solo factor pronóstico ${ }^{15-18}$.

En este estudio, el 36,3 \% de los ACG tratados quirúrgicamente correspondieron a ACGI, datos muy similares a otras series Latinoamericanas. Como consecuencia de un diagnostico tardío, el $85 \%(n=35)$ de los pacientes correspondieron a etapas avanzadas T3 - T4, con lesiones prominentes y con una muy alta proporción de metástasis ganglionares, las cuales se presentaron en el $85 \%$ de los ACGI. Con la excepción de un paciente en el que se reportó un total de ocho ganglios linfáticos analizados, el estudio patológico ganglionar se consideró como satisfactorio, con un promedio de 24 ganglios linfáticos estudiados por espécimen, cumpliendo el estándar de calidad mínimo permisible de 16 ganglios evaluados, lo cual refleja una buena técnica quirúrgica de la linfadenectomía DII, así como una confiable técnica de análisis patológico ${ }^{19,20}$.

Esta serie confirma que las metástasis ganglionares son un fenómeno muy frecuente y esperado en los ACGI avanzados. Estos hallazgos tienen implicaciones terapéuticas, considerando que en el tratamiento de este tipo de lesiones tumorales la cirugía a realizar debe incluir por lo menos una linfadenectomía DII para favorecer un mejor control locorregional y se debe optar por terapias sistémicas complementarias para tener la posibilidad de una mejor supervivencia libre de enfermedad ${ }^{20}$.

\section{Conclusiones}

El adenocarcinoma gástrico indiferenciado es un tumor con características fenotípicas distintivas, que en etapas avanzadas con lesiones T3 - T4 tiene una alta tasa de metástasis ganglionares, en hasta el $85 \%$ de los casos, lo que indica la necesidad de tratamientos quirúrgicos radicales, incluyendo linfadenectomía D II y de terapias sistémicas complementarias adicionales.

\section{Cumplimiento de normas éticas}

Consentimiento informado: Este estudio es una revisión retrospectiva de historias clínicas, y como tal, no hay necesidad de un consentimiento informado. Este estudio contó con la aprobación del Comité de ética institucional del Hospital Militar Central, en Bogotá D.C., Colombia.

Conflicto de interés: Ninguno declarado por los autores.

Fuente de financiación: Financiado con recursos propios de los autores.

\section{Contribución de los autores:}

Concepción y diseño del estudio: Ana María García, William Sánchez.

Adquisición de datos: Ana María García, William Sánchez. Análisis e interpretación de datos: Ana María García, William Sánchez.

Redacción del manuscrito: Ana María García, William Sánchez.

Revisión crítica: Ana María García, William Sánchez.

\section{Referencias}

1. Sánchez W, Abaunza H, Ferreira E, Rasslan S. Cáncer gástrico avanzado. En: Ferreira E, Rassian S, Abaunza $\mathrm{H}$, Ferreira F, editores. Problemas complejos en cirugía abdominal. Editorial Atheneu, Brasil. 2003. p. 29-38.

2. Machlowska J, Maciejewski R, Sitarz R. The pattern of signatures in gastric cáncer prognosis. Int J Mol Sci. 2018;19:1658. https://doi.org/10.3390/ijms19061658

3. Espejo-Romero H, Navarrete-Siancas J. Clasificación de los adenocarcinomas de estómago. Rev. Gastroenterol Peru. 2003;23:199-212.

4. Myeong-Cherl Kook. Risk factors for lymph node metastasis in undifferentiated type gastric carcinoma. Clin Endosc. 2019;52:15-20.

https://doi.org/10.5946/ce.2018.193

5. Choi KK, Bae JM, Kim SM, Sohn TS, Noh JH, Lee JH. et al. The risk of lymph node metastases in 3951 surgically resected mucosal gastric cancer: implications for endoscopic resection. Gastrointest Endosc. 2016;83:896901. https://doi.org/10.1016/j.gie.2015.08.051

6. Sánchez W, Peña D, García D. Mortalidad por cáncer gástrico en Colombia, informe epidemiológico. Rev Colomb Cir. 2008;23(Supl):41.

7. Japanese Gastric Cancer Association. Japanese gastric cancer treatment guidelines 2010. Gastric Cancer. 2011;14:113-23.

https://doi.org/10.1007/s10120-011-0042-4 
8. Nakamura K, Ueyama T, Yao T. Pathology and prognosis of gastric carcinoma. Cancer. 1992;70:1030-7. https://doi.org/10.1002/1097-0142(19920901)70:5 <1030::AID-CNCR2820700504>3.0.CO;2-C

9. Nakamura S, Kato Y. Pathological studies of human gastric cancer. Acta Pathol Jpn 1982;32(Suppl 2):239-47. https://doi.org/10.1002/1097-0142(19920901) 70:5<1030::AID-CNCR2820700504>3.0.CO;2-C

10. Choi YY, Noh SH. Open Surgery for Gastric Cancer: Total Gastrectomy with D2 lymph node dissection. In: Noh SH, Hyung WJ, editors. Surgery for gastric cancer. Springer-Verlag, 2019. p.111-16. https://doi.org/10.1007/978-3-662-45583-8_7

11. Mariette C, Carneiro F, Grabsch HI, Van der Post RS, Allum W. et al. Consensus on the pathological definition and classification of poorly cohesive gastric carcinoma. Gastric Cancer 2019;22:1-9. https://doi.org/10.1007/s10120-018-0868-0

12. Kim YH, Park JH, Park CK, Kim JH, Lee SK, Lee Ych. et al. Histologic purity of signet ring cell carcinoma is a favorable risk factor for lymph metastasis in poorly cohesive, submucosa invasive early gastric carcinoma. Gastric Cancer. 2017;20:583-90. https://doi.org/10.1007/s10120-016-0645-x

13. Luo DH, Zhou Q, Hu SK, Xia YQ, Xu Cc, Lin TS, et al. Differential expression of notch 1 intracelular domain and p21 proteins, and their clinical significance in gastric cancer. Oncol. Lett. 2014;7:471-8. https://doi.org/10.3892/ol.2013.1751

14. Goncalves AR, Carneiro AJ, Martins I, de Faria PA, Ferreira MA, de Mello EL, et al. Prognosis significance of p53 protein expression in early gastric cáncer. Pathol. Oncol. Res.2011;17:349-55.

https://doi.org/10.1007/s12253-010-9333-z

15. Gotoda T, Yanagisawa A, Sasako M, Ono H, Nakanishi Y, Shimoda T. et al. Incidence of lymph node metastasis from early gastric cáncer: estimation with a large number of cases at two large centers. Gastric cancern 2000;3:219-25. https://doi.org/10.1007/PL00011720

16. Ye BD, Kim SG, Lee JY. Predictive factors for lymph node metastasis and endoscopic treatment strategies for undifferentiated early gastric cancer. J Gastroenterol Hepatoln 2008;23:46-50.

https://doi.org/10.1111/j.1440-1746.2006.04791.x

17. Kunisaki C, Takahashi M, Nahahori Y, Fukushima T, Makjino H, Takagawa R. et al. Risk factors for lymph node metastasis in histologically poorly differentiated type early gastric cancer. Endoscopy. 2009;41:498-503. https://doi.org/10.1055/s-0029-1214758

18. Hirasawa T, Gotoda T, Miyata S, Kato Y, Shimoda T, Taniguchi $\mathrm{H}$. et al. Incidence of lymph node metastasis and the feasibility of endoscopic resection for undifferentiated type early gastric cancer. Gastric Cancer. 2009;12:148-52. https://doi.org/10.1007/s10120-009-0515-X

19. Kim WH, Park CK, Kim YB, Kim YW, Kim HG, Bae HI. et al. A standardized pathology report for gastric cancer. Korean J Pathol. 2005;39:106-13.

20. Feng-Hua W, Lin S, Jin L, Zhi-Wei Z, Han L, Xiao T. et al. The Chinese society of clinical oncology (CSCO): clinical guidelines for the diagnosis and treatment of gastric cancer. Cancer Commun. 2019;39:10. https://doi.org/10.1186/s40880-019-0349-9 